Voix et Images

volxetimages

\title{
Platitudes et densités de la poésie contemporaine
}

\section{Luc Bonenfant}

Volume 33, numéro 3 (99), printemps-été 2008

Germaine Guèvremont. Nouvelles survenances

URI : https://id.erudit.org/iderudit/018678ar

DOI : https://doi.org/10.7202/018678ar

Aller au sommaire du numéro

\section{Éditeur(s)}

Université du Québec à Montréal

ISSN

0318-9201 (imprimé)

1705-933X (numérique)

Découvrir la revue

Citer ce compte rendu

Bonenfant, L. (2008). Compte rendu de [Platitudes et densités de la poésie contemporaine]. Voix et Images, 33(3), 124-128.

https://doi.org/10.7202/018678ar d'utilisation que vous pouvez consulter en ligne.

https://apropos.erudit.org/fr/usagers/politique-dutilisation/ 


\title{
P O É S I E
}

Platitudes et densités de la poésie contemporaine

\author{
$+++$ \\ LUC BONENFANT \\ Université du Québec à Montréal
}

Qu'on ne se méprenne pas sur le titre de cette chronique, qui m'est inspiré par un ouvrage récent d'Éric de Chassey consacré à la photographie plate ou bidimensionnelle. «Pour qu'il y ait platitude, écrit Chassey, il faut que la planéité subsiste dans la perception de celui ou de celle qui regarde une image, comme un personnage de dessin animé écrasé par un objet pesant existe un moment à l'état de figure bidimensionnelle, dans une stase plus ou moins longue, avant de soudain, dans un autre temps qui est en fait un autre monde, se redéployer dans l'espace tridimensionnel ${ }^{1}$.»

Les recueils évoqués ici interrogent chacun à leur manière une facette de cette question. Détournée de l'acception iconographique que lui donne Chassey, la platitude engage des modes de dérivation du poétique: la typographie de la page (chez Simon Philippe Turcot), les hypothétiques linéarités de la mémoire et du paysage (chez Guillaume Lebel) ou, encore, la compression du langage aphasique (chez Philippe More) constituent autant de points de butée permettant à la voix poétique de faire advenir les principes de nouvelles économies de sens.

Le paysage est un atelier ${ }^{2}$ de Simon Philippe Turcot est porté par une grande exigence. Le lecteur pressé croira d'abord au simple déploiement des couleurs. En effet, les tons les plus vifs, tels le rouge de l'«artère de Naples » (14) ou le "jaune aveugle» de «quelques citronniers» (14), pétillent entre la «nuit blanche suspendue» (11) et la «ligne horizontale turquoise» (13). Mais ils s'inscrivent aussi dans un jeu de perspectives qui déploient pleinement les poèmes-tableaux de Turcot. Ainsi, les «fines lignes verticales et noires/jusqu'au sommet du Vésuve/ou du ciel pâle» (16) ouvrent un horizon où la parole se forgera désormais dans la tridimensionnalité du monde qu'elle donne à lire.

1 Éric de Chassey, Platitudes. Une histoire de la photographie plate, Paris, Gallimard, coll. "Arts et artistes", 2006, p. 12. 2 Simon Philippe Turcot, Le paysage est un atelier, Montréal, Les heures bleues, coll. «Le dire», 2007, 63 p. 
Il va de soi que l'écriture doit composer avec la planéité de cet objet bidimensionnel qu'est le livre. La profondeur optique appartient d'emblée aux arts plastiques (peinture, photographie, cinéma, télévision), et les tentatives poétiques pour occuper l'espace de la page ont surtout conçu l'étalement dans les marges de son cadre $^{3}$. Placés de manière éparse sur la page, les vers libres de Turcot investissent pourtant bel et bien l'impossible troisième dimension du Livre. Chez Turcot, l'atelier poétique intègre pleinement la leçon plastique.

$996 \mathrm{~km} / \mathrm{h}$

transatlantique

nuit blanche suspendue

en bas le jour se lève

sur une Irlande inconnue (11).

Disposé en forme d'escalier - ou imitant le geste de l'avion qui s'apprête à atterrir - ce poème liminaire reproduit tout à la fois la densité du temps qui fuit vers l'avant et la profondeur verticale d'un espace envisagé à partir de l'altitude du jet transatlantique. La platitude typographique permet de montrer le monde plutôt que de seulement le dire. Ailleurs, des sortes de didascalies, mises entre parenthèses sur la page, détournent la linéarité des mots en suggérant la profondeur éventuelle appelée par un projet qui reste résolument multidisciplinaire: «au moins trois mille oiseaux/(tableau)/à fendre un violon» (17). Une telle intégration de la perspective spatiale dans le cadre livresque est assez rare dans le champ poétique québécois contemporain, où le vers libre est le plus souvent disposé depuis la marge de gauche. Elle ne sera pas non plus aisée: écrit en diptyque, le recueil compose le fil d'un apprentissage où le poète veut «transpercer l'ailleurs/d'un battement de cils» (28), c'est-à-dire comprendre le monde à partir du regard qu'il pose sur lui.

Le voyage vers l'Europe, thème central de la première partie, apporte rapidement sa violence quand l'arrivée dans la ville de Český Krumlov marque une sorte d'impossibilité du projet visuel: ce joyau architectural du patrimoine mondial happe le poète en lui montrant plutôt la réalité sonore et musicale de l'univers des gitans qui l'habitent. Dans la seconde partie du recueil, celle du retour en terre québécoise, le poète devra conséquemment «réapprendre le mélange des couleurs» (39). La perspective du regard s'y trouve «en jachère (42) et «les profondeurs ne sont plus sondées» (47). Mais porté par l'idéal fantasmagorique de la troisième dimension du Livre, le poète acceptera finalement de "peindre ce qui est/désormais possible» (50). Les derniers poèmes de la section verront donc le retour des perspectives et des

3 Malgré sa modernité essentielle, même le Coup de dés ne laisse jamais oublier sa platitude: son rythme appartient d'emblée au "papier [qui] intervient chaque fois qu'une image, d'elle-même, cesse ou rentre, acceptant la succession d'autres». Stéphane Mallarmé, "Préface de l'édition "Cosmopolis" ", Igitur, Divagations. Un coup de dés, Paris, Gallimard, coll. «Poésie», 2003, p. 442. 
couleurs franches quand «au matin les potagers/suant l'huile de lin/réveillent les tons/de vert» (59). Répondant pleinement à son épigraphe, "Je peins pour parler comme j'écris pour voir. RoLAND GIGUĖRE», Le paysage est un atelier permet que peinture et littérature dialoguent franchement, sans complaisance aucune.

«[O]n prend du papier blanc/on y trace un cercle/d'encre blanche/ou d'eau.» (36) Ces vers de La voix meurt pleine ${ }^{4}$, de Guillaume Lebel, accomplissent la platitude picturale parfaite puisque la trace blanche du crayon se fond littéralement dans le blanc du papier ${ }^{5}$. Subsiste une page en apparence vierge, comme si la mémoire scripturale ne réussissait pas à s'y inscrire de façon durable. La transparence du procédé constitue le point d'ancrage de cette épaisseur paradoxale qui traverse tout le recueil et que la deuxième et dernière strophe du poème donne justement à lire: «chaque jour/on dilue ainsi ce qu'il reste de nous-mêmes» (36). L'écriture de la voix surgit très exactement de la possibilité advenue d'une parole entièrement faite d'anfractuosités et de manques. Elle est, de ce point de vue, notre contemporaine la plus immédiate: engagée dans l'impossibilité de son unité, dévolue à ses inachèvements et à ses discordances. Toujours arrivée «au seuil d'une nouvelle incomplétude» (61), cette voix accède à une fragilité qui l'oblige à la dissociation la plus totale quand «quelqu'un dans nos pas/on se regarde partir/hors de nos os» (41).

On aura remarqué la désarticulation lexicale de cette voix, que les pronoms «Je», «nous» et "on » semblent successivement désigner. Paradoxalement, le récit de cette perte - perte de soi ? perte de l'autre? perte des autres? — indique le désir impérieux d'exister dans un monde qui semble toujours vouloir s'échapper tandis qu'« on pose la main sur une fenêtre/voulant prendre appui sur le monde/mais les arbres tombés/ont tiré l'horizon avec eux » (52). S'ils rappellent inévitablement ceux de Loranger ${ }^{6}$, ces vers ne disent pas pour autant l'immobilité d'un regard appuyé ; ils portent en eux le désir d'un secours, celui du monde qui s'accorderait au sujet plutôt que de s'y dérober. La voix poétique, d'ailleurs, erre et se déplace tout au long du recueil, tente un mouvement qui confirmera constamment son existence. Exit le repli pessimiste. Le ton des vers, ici, n'est ni sombre, ni tragique, ni menaçant.

Je dirais même que La voix meurt pleine est entièrement traversée par une luminosité qui, pour problématique qu'elle soit, n'en reste pas moins bien réelle: quand «le mur s'écroule/la lumière apparaît» (13). L'oxymore du titre aura donc dit bien des choses: la voix meurt pleine justement parce que la beauté surgit inopinément quand «l'éclaboussure de l'abandon/fait une fleur de sang dans nos yeux »

4 Guillaume Lebel, La voix meurt pleine, Montréal, l'Hexagone, coll. «L'appel des mots», 2007.5 «La seule photographie complètement et littéralement plate de toute l'histoire est le photogramme Noir sur noir réalisé tardivement (vers 1945-1946) par Alexander Rodtchenko.» Éric de Chassey, op. cit., p. 11.6 "Je regarde dehors par la fenêtre./J'appuie des deux mains et du front sur la vitre./Ainsi, je touche le paysage,/Je touche ce que je vois,/Ce que je vois donne l'équilibre/Ā tout mon être qui s'y appuie./[...] Tout mon être appuyé au dehors solidarisé. » Jean-Aubert Loranger, Les atmosphères, Montréal, L.A. Morissette, 1920, p. 38. 
(47). L'écriture a ici besoin du legs des vestiges et des peurs anciennes, parce que "pour exister/[...] on écrit/on se sent vide» (29). La blancheur de cette voix privée de sa plénitude instaure le nécessaire rapport d'une écriture qui va vers elle-même dans les fragments de son parcours. Refusant l'horizontalité étale du paysage qui se trouve «là : à bout de bras» (15), la voix oscille entre les segments de la route où elle se trouve. «je regarde où je vais/d'où j'arrive/il n'y a plus de destination » (14), ditelle, comme pour montrer la valse-hésitation où l'anticipation et la prospection semblent la contenir.

Le tout dernier poème du recueil l'indique bien: c'est finalement dans la brèche des ruines que la voix trouvera le lieu de son nouveau départ: "une brèche se creuse où s'arrêtent nos ruines/je pars/je te lègue l'index de quelqu'un/une voix qui n'est pas tracée d'avance/j'amène le reste du corps » (74). Il ne s'agit pas d'oublier le passé mais plutôt de le porter avec soi, tel le reste de ce corps, de manière à l'articuler aux débris de sa mémoire. Détournés de l'horizontalité plate, le temps et le paysage engendrent la condition nécessaire de l'existence d'une voix qui ne se sera jamais mieux rencontrée que dans ce qu'elle contient de moins assuré.

Aussi peu assurées sont les voix qui construisent le dialogue implicite de Nos images $d^{\prime}$ aphasie $^{7}$, de Philippe More, où la quotidienne abdication dit en quelque sorte la nécessaire disparition élocutoire de celle qui aurait dû être le sujet des textes: «Ne parle plus/qu'au seuil tremblé de ta présence, écris/à la faveur fantôme d'une quotidienne abdication.» (31) Nécessaire disparition, parce que l'aphasie qu'on sent bien réelle, presque médicale dans la mesure où Philippe More pratique aussi le métier d'urgentologue, recouvre une aphasie plus vastement symbolique où la parole - quelle qu'elle soit - appelle toujours déjà les psalmodies du silence.

Nous sommes habitués à lire aujourd'hui du vers libre défait de toute ponctuation. Contrairement à cela, les deux-points et les virgules, mais aussi les points d'interrogation, abondent dans les vers libres de la première partie de ce recueil où l'aphasie ne touche pas tant la syntaxe et la grammaire que la dimension plus proprement lexicale du langage, ouvrant du coup le pouvoir d'évocation des images les plus troublantes: «Nul oxygène où quelque soir veiller,/fond maigre de prière où s'articulerait/l'éternité infinitive de rêver à court d'images:/ailleurs, la vieille manie d'exister, ailleurs.» (14) La ponctuation régule les phrases qui échapperaient autrement à toute logique discursive. Le discours découvre le décalage bien réel qui existe entre les aberrations lexicales de l'aphasique et les encadrements uniformisants de la syntaxe régulière de celui qui parle à sa place.

Cette rencontre de l'autre au sein même de son langage semble être l'occasion pour le sujet de s'initier à sa propre absence. D'autres mots remplaceront l'ancienne parole, même s'ils ne semblent pas plus assurés que les premiers: "pas de mémoire

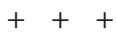

7 Philippe More, Nos images d'aphasie, Montréal, Poètes de brousse, 2007, 69 p. 
où reguérir d'avoir parlé,/d'avoir presque inventé entre les ondes/la moindre épaisseur d'homme » (35). Spectrale, la voix parle depuis le lieu de sa mort symbolique et l'adhésion à la parole aphasique appelle une communion où seule l'expérience intérieure rendra finalement compte de l'être: «l'ordre douloureux que dans les mots/ton corps eut à inscrire, demeurent encore/ce paysage à court d'absence,/cette manière d'envahir par l'intérieur/volumes, coloris et dimensions» (23). Qu'on ne se méprenne pas, l'espérance est ici au rendez-vous quand surgit, dans la deuxième partie, une parole nouvelle dont l'inscription cosmique tranche avec le ton plus sombre et recueilli de la première partie. Comme si l'aphasie était surtout le lieu d'une liberté, celle d'une "histoire à l'échelle des anges» (57). Dès lors qu'il est investi d'un espoir, le récit du deuil d'aphasie mène donc à une nouvelle possibilité.

La seconde partie du recueil gomme d'ailleurs le fonctionnement syntaxique de la ponctuation des premiers poèmes pour laisser place à des strophes plus courtes, généralement de deux à cinq vers (une strophe fait exception; elle contient six vers), séparées les unes des autres par un astérisque. Libérée des contraintes du discours plat, la parole aphasique se trouve allégée et son mode de figuration textuelle est désormais celui des bribes qui la construise. La platitude de la syntaxe ordinaire se dérobe sous une dimension où le sujet peut se déployer dans le «rythme arrondi du monde/éclos ailleurs avec les yeux/et pendant ce temps le toucher/qui exerce de loin/ses petits aveuglements d'étoile» (54).

Pourtant portés par des thèmes archiconnus - le rapport aux arts, l'existence lyrique, l'impossibilité du dire - ces recueils n'ont rien à voir avec la banalité d'un discours convenu ou des clichés ressassés. Ils engagent chacun à leur façon une réflexion critique, œuvrant de la sorte à une offrande réelle et diligente de la parole. En s'ouvrant ainsi au malaise langagier nécessaire à la posture poétique, leurs trois auteurs disent qu'ils sont convaincus de sa nécessité parce qu'il faut, comme l'écrit More, «surprendre dans le texte un sortilège/ma propre imprégnation dans les pages perdues» (68). 